\title{
Monte Carlo Simulation of a Submicron MOSFET Including Inversion Layer Quantization
}

\author{
J.B. ROLDAN*, F. GAMIZ, J.A. LOPEZ-VILLANUEVA and J.E. CARCELLER \\ Departamento de Electrónica y Tecnología de Computadore, Universidad de Granada, Facultad de Ciencias, Avd.Fuentenueva s/n.
} 18071 Granada, Spain

\begin{abstract}
A Monte Carlo simulator of the electron dynamics in the channel, coupled with a solution of the two-dimensional Poisson equation including inversion-layer quantization and drift-diffusion equations has been developed. This simulator has been applied to the study of electron transport in normal operation conditions for different submicron channel length devices. Some interesting non-local effects such as electron velocity overshoot can be observed.
\end{abstract}

\section{INTRODUCTION}

Non-local effects are becoming more and more prominent as MOSFET dimensions shrink to deep-submicrometer regimes. In order to study these effects, nonlocal models must be used to accurately describe electron transport in MOSFETs. The Monte Carlo method is held to contain a more rigorous description of device physics than models based on the solution of fundamental balance equations [1-2]. A quantum description of the confined inversion layer should also be included [3-4]. Knowledge of electron mobility dependence on variables such as the transverse and longitudinal electric fields is important for simulation and modelling of MOSFETs. Monte Carlo simulations are essential to study these electron mobility dependencies needed for circuit simulators of state-of-the-art MOSFETs.

\section{DEVICE SIMULATION}

We have simplified the solution of all the equations involved in the description of electron transport in a submicron MOSFET with the procedure described below. To have a departure point we took the following steps: The quasifermi levels as well as their separation were assumed to be constant in the transverse direction but were allowed to vary along the longitudinal direction. The total variation of the quasifermi levels along the channel is given by the drain-tosource bias voltage and is broken down into a sequence of steps spread out among several still undefined spatial locations along the channel. According to this hypothesis, the device is divided (by setting $\mathrm{N}-1$ points inside the channel) into an appropriate number $\mathrm{N}$ of smaller channels (subchannels) of unknown length $\mathrm{L}_{\mathrm{i}}$, where $\Sigma \mathrm{L}_{\mathrm{i}}=\mathrm{L}, \mathrm{L}$ is the effective channel length. The one-dimensional Schroedinger and Poisson equations are then selfconsistently solved at both ends of each subchannel, taking into account the pseudofermi level separation at each point. Each subchannel is then described as a sheet layer of charge located inside the semiconductor bulk at a distance $z_{I}$ from the silicon-oxide interface. This position $\mathrm{z}_{\mathrm{I}}$ is the mean transverse position of the inversion electron dis-

\footnotetext{
* Corresponding author. Tel: 34-58-243227. Fax: 34-58-243230. Email: paco@gcd.ugr.es or jroldan@goliat.ugr.es
} 
tribution. The current in the subchannel is obtained by adding drift and diffusion contributions. If the voltage drop across the subchannel is very small, the following expression for the drain current $\left(\mathrm{I}_{\mathrm{DS}}\right)$ is obtained:

$$
\begin{aligned}
\mathrm{I}_{\mathrm{DS}}=\frac{\mathrm{qW} \mu_{\mathrm{i}}\left(\mathrm{E}_{\|}, \mathrm{E}_{\perp}\right)}{\mathrm{L}_{\mathrm{i}}}\left[-\overline{\mathrm{N}}_{\mathrm{I}}\left(\Psi_{\text {drain }}^{*}-\Psi_{\text {source }}^{*}\right)\right. \\
\left.+\phi_{\mathrm{t}}\left(\mathrm{N}_{\mathrm{I}, \text { drain }}-\mathrm{N}_{\mathrm{l}, \text { source }}\right)\right]
\end{aligned}
$$

where $\psi^{*}$ is the electrostatic potential in $\mathrm{z}_{\mathrm{I}}, \mathrm{N}_{\mathrm{I}}$ the electron density in the subchannel ends, $N_{I}$ the average density of electrons in the subchannel, and $\phi_{t}$ the thermal voltage. The electron mobility depends on both the longitudinal $\left(\mathrm{E}_{\mathrm{II}}\right)$ and transverse $(\mathrm{E} \perp)$ electric fields. An approximated dependence on $\mathrm{E}_{\mathrm{II}}$ is assumed for the first time, while an accurate expression obtained by one-electron Monte Carlo simulation is used for the low-field mobility and its dependencies with the transverse-electric field and the temperature, which is crucial for reproducing with accuracy experimental results. The length of each subchannel is obtained by applying Expression 1 according to an iterative procedure.The two-dimensional Poisson equation is solved using the solution obtained previously as a starting point :

$$
\frac{\partial^{2} \phi(x, y)}{\partial x^{2}}+\frac{\partial^{2} \phi(x, y)}{\partial y^{2}}=-\frac{\rho(x, y)}{\varepsilon_{s}}
$$

where " $x$ " is the parallel and " $y$ " the perpendicular co-ordinates to the channel. An adaptive grid has been set in the whole structure. The two-dimensional problem considered in (2) has been decomposed into $\mathrm{N}$ one-dimensional problems :

$$
\frac{\mathrm{d}^{2} \phi_{\mathrm{i}}(\mathrm{y})}{\mathrm{dy}^{2}}=-\frac{\widetilde{\rho}_{\mathrm{i}}(\mathrm{y})}{\varepsilon_{s}}
$$

where

$$
\tilde{\rho}_{i}(y)=\rho\left(x_{i}, y\right)+\left.\varepsilon_{s} \frac{\partial^{2} \phi_{0}(x, y)}{\partial x^{2}}\right|_{x=x_{i}}
$$

$\Phi_{\mathrm{o}}(\mathrm{x}, \mathrm{y})$ is the solution obtained previously, and $\mathrm{x}_{\mathrm{i}}$ is a grid column in the channel. With this effective charge density $\tilde{\rho}_{\mathrm{i}}(\mathrm{y})$, we can use the procedure explained above to solve the Poisson and Schroedinger equations, repeated until a convergence criterium is reached.
Once the actual potential distribution, longitudinal and transverse fields, and inversion and depletion charge concentrations along the channel have been calculated, the electron dynamics are simulated by the Monte Carlo method. The grid is chosen to be thin enough that a constant value of the different transport magnitudes can be assumed in each grid interval (i.e. each grid interval was characterised by a constant value of the longitudinal- and transverse-electric fields, surface potential, inversion and depletioncharges, electronic subband minima, etc.). Taking into account these values, the scattering rates are evaluated in each grid zone. Phonon, surface-roughness, and Coulomb scattering have been considered, following the procedure in a previous work [4-6]. To continue in Monte Carlo simulation, a great number of electrons are introduced, one by one, into the channel from the source. The longitudinal electric field in each grid zone modifies the electron wavevector according to the semiclassical model during a free flight whose length is calculated according to a standard Monte Carlo procedure by generating a random number and using the maximum of the total scattering rate along the whole channel. The time an electron spends in each grid zone, the electron mean-velocity and mean-energy in each interval are recorded. The electron velocity distribution along the channel can thus be evaluated. Taking into account expression $\mathbf{v}_{\text {drift }}=\mu(\mathbf{x}) \mathbf{E}_{\text {II }}$ we can define a local electron mobility in the channel to be used in Expression 1 to calculate the new drain current and subchannel lengths. The whole procedure is solved again until a convergence criterium is reached.

\section{RESULTS}

$0.1 \mu \mathrm{m}, 0.2 \mu \mathrm{m}, 0.25 \mu \mathrm{m}, 0.5 \mu \mathrm{m}$, and $1 \mu \mathrm{m}$ channel length MOSFETs have been simulated. The bulk doping concentration was $\mathrm{N}_{\mathrm{A}}=4 \times 10^{17} \mathrm{~cm}^{-3}$, the gateoxide thickness $\mathrm{t}_{\mathrm{ox}}=5.6 \mathrm{~nm}$ and the source junction depth $x_{j}=0.1 \mu \mathrm{m}$. Figures 1 and 2 show the $2 \mathrm{D}$ electrostatic potential distribution and the $3 \mathrm{D}$ electron concentration for the $0.2 \mu \mathrm{m}$ channel MOSFET simulated for $\mathrm{V}_{\mathrm{GS}}=2.4 \mathrm{~V}$ and $\mathrm{V}_{\mathrm{DS}}=1 \mathrm{~V}$. The short-chan- 


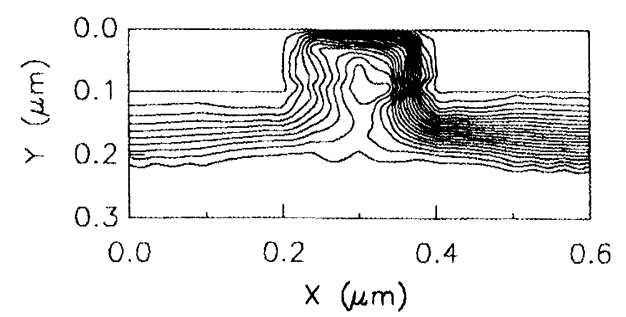

FIGURE $12 \mathrm{D}$ electrostatic potential plot for the MOSFET simulated. $\mathrm{V}_{\mathrm{GS}}=2.4 \mathrm{~V}, \mathrm{~V}_{\mathrm{DS}}=1 \mathrm{~V}, \mathrm{x}_{\mathrm{j}}=0,1 \mu \mathrm{m}, \mathrm{N}_{\mathrm{A}}=4 \times 10^{17} \mathrm{~cm}^{-3}$, $\mathrm{t}_{\mathrm{ox}}=5.6 \mathrm{~nm}, \mathrm{~L}_{\mathrm{eff}}=0.2 \mu \mathrm{m}$

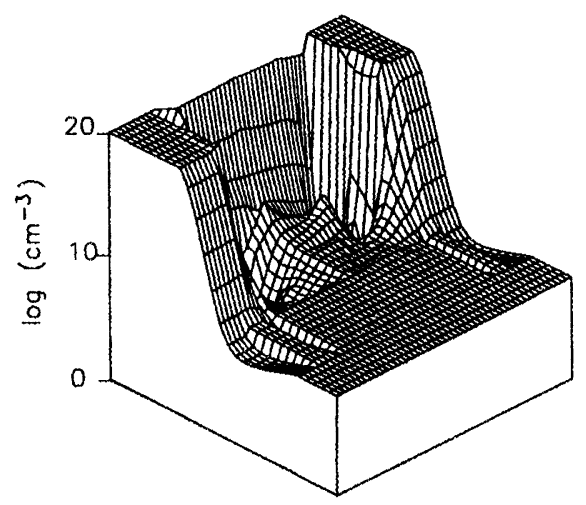

FIGURE 2 3D electron density distribution plot for the MOSFET simulated. $\mathrm{V}_{\mathrm{GS}}=2.4 \mathrm{~V}, \mathrm{~V}_{\mathrm{DS}}=1 \mathrm{~V}, \mathrm{x}_{\mathrm{j}}=0.1 \mu \mathrm{m}, \mathrm{N}_{\mathrm{A}}=4 \times 10^{17} \mathrm{~cm}^{-3}$, $\mathrm{t}_{\mathrm{ox}}=5.6 \mathrm{~nm}, \mathrm{~L}_{\mathrm{eff}}=0.2 \mu \mathrm{m}$

nel effects are quite evident even for this bias; however, we have seen that the two-dimensional current corrections to the one-dimensional model are not important due to the high bulk-doping concentration. The average kinetic-energy distribution for electrons along the channel is always below $0.5 \mathrm{eV}$, in the range of external voltages covered in this work. Therefore, according to Laux and Fischetti [1], a simplified description of the silicon band structure is justified. Nevertheless, we have also represented the effects of non-parabolicity on the electron dynamics in the channel. The inversion charge distribution along the channel is shown in Figure 4, Monte Carlo results (symbols) and drift-diffusion results (solid line) are compared. Both curves are in good agreement along the channel, and the same agreement was observed for the rest of transistors simulated. Figure 3 shows

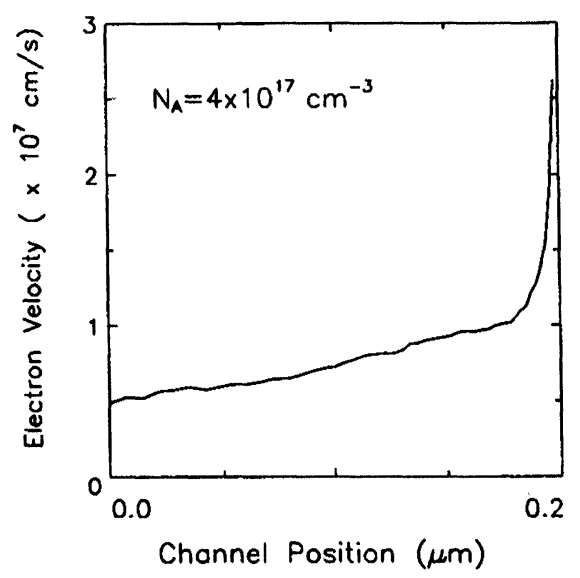

FIGURE 3 Electron velocity versus channel position. Electron velocity overshoot can be observed near the drain. $\mathrm{V}_{\mathrm{GS}}=2.4 \mathrm{~V}$, $\mathrm{V}_{\mathrm{DS}}=1 \mathrm{~V}, \quad \mathrm{x}_{\mathrm{j}}=0.1 \mu \mathrm{m}, \quad \mathrm{N}_{\mathrm{A}}=4 \times 10^{17} \mathrm{~cm}^{-3}, \quad \mathrm{t}_{\mathrm{ox}}=5.6 \mathrm{~nm}$, $\mathrm{L}_{\text {eff }}=0.2 \mu \mathrm{m}$

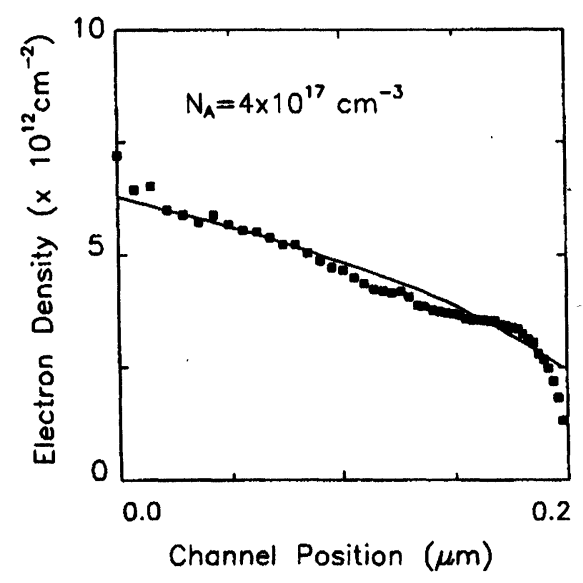

FIGURE 4 Electron density along channel obtained by Monte Carlo method (symbols) and by drift-diffusion method (solid line). $\mathrm{V}_{\mathrm{GS}}=2.4 \mathrm{~V}, \quad \mathrm{~V}_{\mathrm{DS}}=1 \mathrm{~V}, \quad \mathrm{x}_{\mathrm{j}}=0.1 \mu \mathrm{m}, \quad \mathrm{N}_{\mathrm{A}}=4 \times 10^{17} \mathrm{~cm}^{-3}$, $\mathrm{t}_{\mathrm{ox}}=5.6 \mathrm{~nm}, \mathrm{~L}_{\text {eff }}=0.2 \mu \mathrm{m}$

the electron drift velocity along the channel obtained by the MC method. Noticeable velocity overshoot can be observed near the drain. Fig 5 shows the electron mobility along the channel for the same MOSFET. Making use of the Monte Carlo results obtained we are able to model electron mobility accounting for electron velocity overshoot and other effects observed in very short channel length MOSFETs and use them in simple drift-diffusion simulators. 


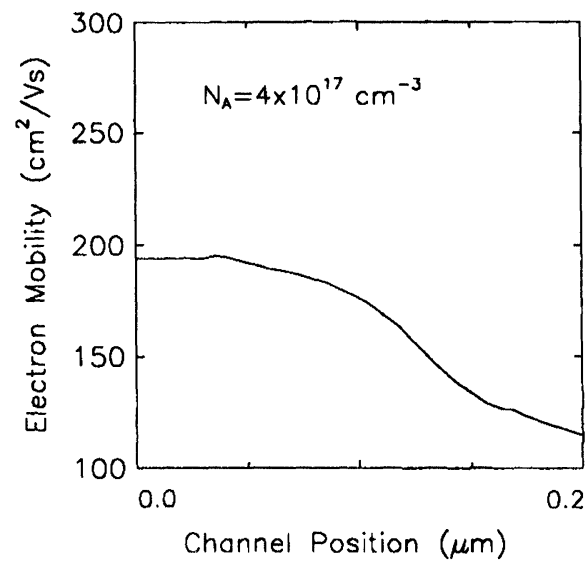

FIGURE 5 Electron mobility versus channel position. $\mathrm{V}_{\mathrm{GS}}=2.4 \mathrm{~V}$, $\mathrm{V}_{\mathrm{DS}}=1 \mathrm{~V}, \mathrm{x}_{\mathrm{j}}=0.1 \mu \mathrm{m}, \mathrm{N}_{\mathrm{A}}=4 \times 10^{17} \mathrm{~cm}^{-3}, \mathrm{t}_{\mathrm{ox}}=5.6 \mathrm{~nm}$, $\mathrm{L}_{\text {eff }}=0.2 \mu \mathrm{m}$

\section{CONCLUSION}

The study of non-local effects such as velocity overshoot, as well as modelling of the dependence of electron mobility and other magnitudes needed to describe electron transport in a MOSFET channel on the transverse and longitudinal electric fields along the channel, has been made possible using a Monte Carlo simulator of the electron dynamics in the channel, coupled with a solution of the two-dimensional Poisson equation including inversion-layer quantization and drift-diffusion equations.

\section{References}

[1] S.E. Laux, M.V. Fischetti, "Monte Carlo simulation of submicrometer Si N-MOSFET's at 77 and 300 K", IEEE Electron Device Lett., 9, p. 467 Sep. (1988)

[2] G. Baccarani, M.R. Wordeman, "An investigation of steadystate velocity overshoot in silicon", Solid-State Electron., 28, p. 407 (1985)

[3] M.V. Fischetti, S.E. Laux, "Monte Carlo study of Electron Transport in Silicon Inversion Layers" Physical Review B, 48, p. 2244 (1993)

[4] F. Gamiz, J.A. Lopez-Villanueva, J.A. Jimenez-Tejada, I. Melchor, and A. Palma, "A Comprehensive Model for Coulomb scattering in inversion layers", Journal Of Applied Physics, 75(2), p. 924 (1994)

[5] F. Gamiz, J.A. Lopez-Villanueva, J. Banqueri, J.E. Carceller, and P. Cartujo., "Universality of Electron Mobility Curves in MOSFETs: A Monte Carlo study" IEEE Trans. Electr. Devices, ED-42, p. 258, (1995)

[6] F. Gamiz, J.A. Lopez-Villanueva, J. Banqueri, Y. Ghailan, and J.E. Carceller, "Oxide Charge Space Correlation in inversion layers II. Three-dimensional oxide charge distribution.", Semicond. Sci. Technol. 10, p. 592-600 (1995)

\section{Biographies}

Juan B. Roldan graduated with a degree in physics in 1993 at Granada University. Since 1993 he has been working on the MOS device physics including 2D transport, non-local effects, electron mobility dependencies on transverse and longitudinal electric fields by using a Monte Carlo MOSFET simulator. $\mathrm{He}$ is a Teaching Assistant at the University of Granada.

Francisco Gamiz graduated with a degree in physics in 1991, and received the Ph.D. in 1994 from the University of Granada. Since 1991 he has been working on the characterization of scattering mechanisms and their influence on the transport properties of charge carriers in semiconductor structures. He has studied electron mobility in silicon inversion layers by Monte Carlo method. His current research interest includes the effects of many-carriers on the electron mobility and the theoretical interpretation of the influence of high longitudinal electric fields have on the electric properties of MO transistors. He is an Associate Professor at the University of Granada.

Juan A. Lopez-Villanueva graduated with a degree in physics in 1984, and received the $\mathrm{Ph}$. $\mathrm{D}$. in 1990 from the University of Granada with a thesis on the degradation of MOS structures by Fowler-Nordheim tunneling. Since 1990 he has been working on the MOS device physics including 2D transport, effects of nonparabolitity, quantum effects, scattering mechanisms and Monte Carlo simulation of charge transport. His current research interest includes the characterization, simulation and modelling of electron devices. He is an Associate Professor at the University of Granada.

Juan E. Carceller graduated with a degree in physics in 1975, and received the $\mathrm{Ph}$. D. degree in 1979 from the University of Barcelona. He has engaged in the research and characterization of deep levels in semiconductors. His current research interest includes degradation of MOS structures and characterization of electron mobility in the channel of MOS transistors. He has been a Professor at the Universities of Barcelona and Granada. 

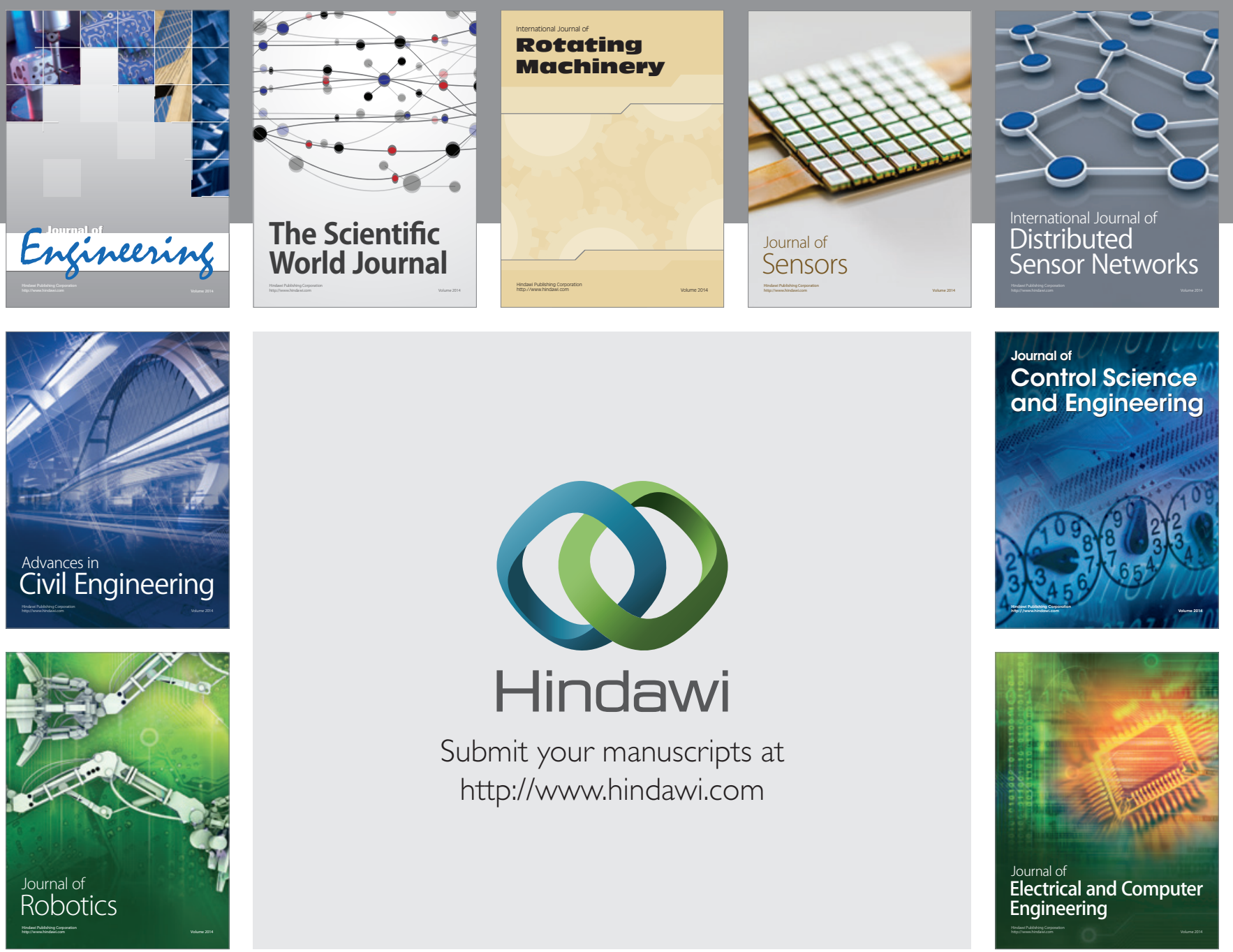

Submit your manuscripts at

http://www.hindawi.com
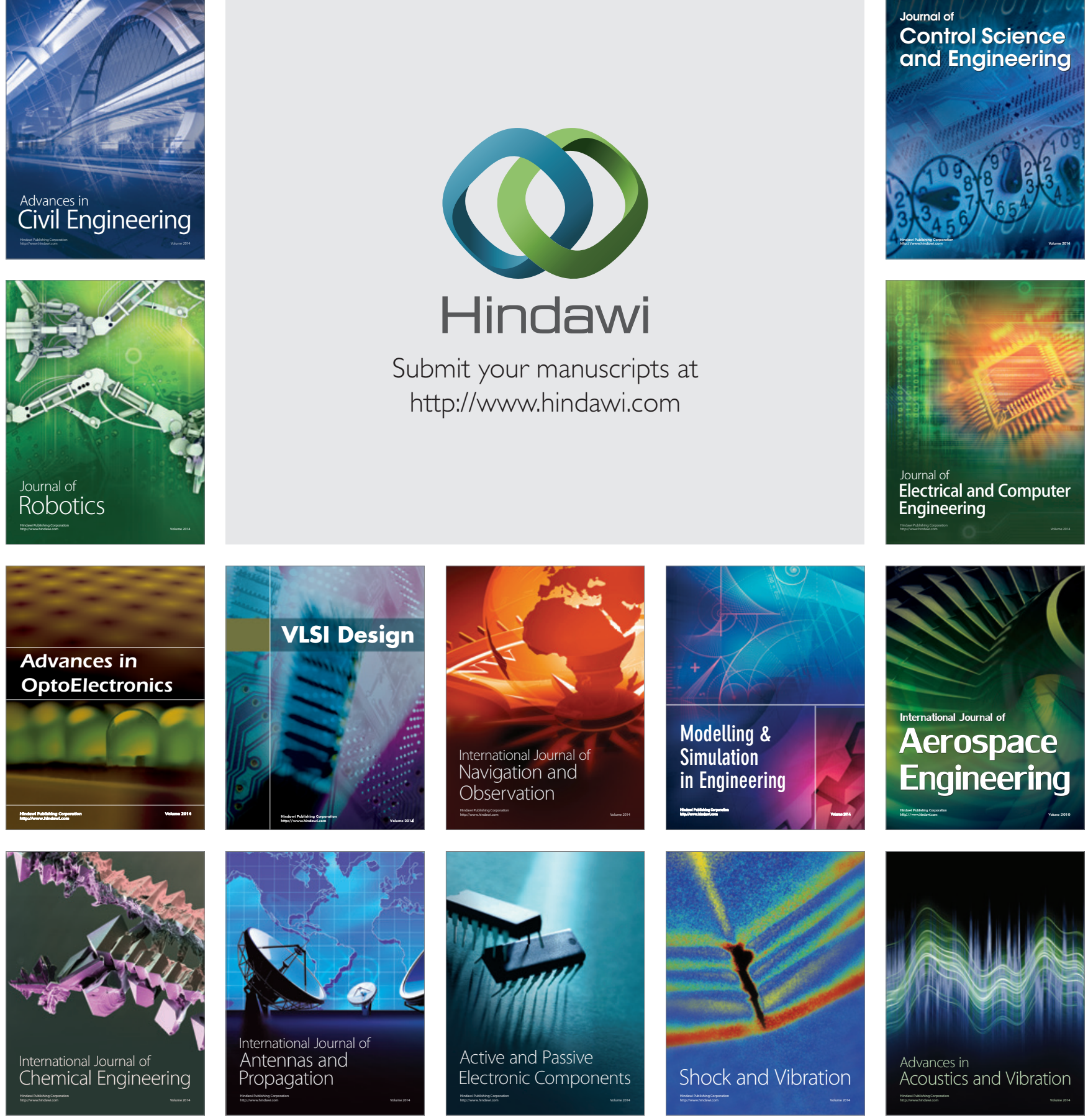